\title{
Low-dose CT-derived attenuation scan: One acquisition, more applications?
}

\author{
Riemer H. J. A. Slart, MD, PhD, ${ }^{\mathrm{a}, \mathrm{b}}$ and Michel Koole, $\mathrm{PhD}^{\mathrm{a}, \mathrm{c}}$ \\ a Department of Nuclear Medicine and Molecular Imaging, University Medical Center Groningen, \\ University of Groningen, Groningen, The Netherlands \\ ${ }^{b}$ Faculty of Science and Technology, University of Twente, Enschede, The Netherlands \\ c Department of Nuclear Medicine, University Hospital Leuven, Leuven, Belgium
}

Received Oct 24, 2014; accepted Oct 25, 2014

doi: $10.1007 / \mathrm{s} 12350-014-0029-8$

\section{See related article, pp. 419-428}

Coronary artery calcium (CAC) is an important marker of coronary artery disease and is of prognostic value for cardiovascular events. ${ }^{1}$ Usually, CAC is measured using a dedicated high-dose CT scanning protocol. However, in case of hybrid PET/CT imaging, CT imaging is often limited to low-dose CT scanning only to correct the PET data for the absorbing effect of the adjacent tissue and to reconstruct attenuation corrected (CTAC) emission data.

Myocardial perfusion imaging (MPI) using PET-CT technology requires a CT scan for attenuation correction but is not used routinely to measure the coronary calcium burden. In general, two CTAC scans are performed to correct for rest and stress MPI. To reduce the number of CTAC scans necessary for MPI and CAC quantification Kaster et al, ${ }^{2}$ evaluated in the current issue of the journal: (1) if a low-dose CTAC scan can also accurately quantify CAC, (2) one low-dose CTAC scan can be used for both the rest MPI as well the stress MPI.

They evaluated in a standard cohort of patients the post-stress CT attenuation scan (stress CTAC), using the same settings as for the rest CTAC scan. In the validation cohort, a modified post-stress CT attenuation scan (Ca-CTAC) was acquired to measure CAC. This modified scan protocol was a similar low-dose, cine-mode,

Reprint requests: Riemer H. J. A. Slart, MD, PhD, Department of Nuclear Medicine and Molecular Imaging, University Medical Center Groningen, University of Groningen, P.O. Box 30001, Hanzeplein 1, 9700 RB, Groningen, The Netherlands; r.h.j.a.slart@umcg.nl J Nucl Cardiol 2015;22:429-30.

$1071-3581 / \$ 34.00$

Copyright (C) 2014 American Society of Nuclear Cardiology. prospective ECG-gated CT scan, acquired at end-expiration breath-hold, and at $70 \%$ of the cardiac phase with two times the standard-CTAC $\mathrm{mA}$.

The major findings were that a modified Ca-CTAC scan showed a trend towards slight decreases of $2 \%$ $3.5 \%$ in segmental stress perfusion in the anterior wall segments $(p<.05)$.

Correlation and agreement between the proposed Ca-CTAC and standard CAC-CT calcium scores at the optimal threshold of $110 \mathrm{HU}$ were also excellent $\left(r^{2}=0.99, \kappa=1.0\right)$. There was a small bias in the regression slope vs unity: $\mathrm{Ca}-\mathrm{CTAC}=0.96 \times \mathrm{CAC}$ $(p<.05)$, but the categorical classification of calcium was accurate in all 23 patients $(\kappa=1.0)$. It was concluded that a single low-dose rest CTAC scan can be used for accurate attenuation correction of both rest and stress PET perfusion images, thus allowing a post-stress CTAC scan to quantify CAC. This is particularly important since it only uses one-quarter of the radiation dose of the standard CAC-CT. This scan also detects the presence/absence of calcium with $100 \%$ accuracy. On the other hand, increased respiratory- and heart-rate adversely affect the ability to co-register the stress PET and post-stress CTAC scans. In this study, however, this was observed in only one patient suggesting that rest CTCTAC may actually be more accurate than stress CTAC for the reconstruction of stress PET perfusion images. As mentioned by the authors, a potential limitation of the present study is that the PET reconstruction assumes an end-expiration CT scans for proper attenuation correction. If the CT scan is not performed properly, a motion artifact can be introduced into the reconstructed perfusion images as observed in the one patient, which was excluded. Careful quality assurance is therefore critical to ensure that PET and CTAC scans are properly acquired and aligned. Alternative use of the proposed Ca-CTAC protocol may be considered before the rest PET scan, instead of post-stress as evaluated in the 
present study. Lower heart-rates prior to pharmacological stress are expected to result in less CT motion artifacts. In a previous study, a rest CTAC scan was performed at a lower $\mathrm{HU}$ threshold to measure coronary calcium and achieved a $\kappa=0.72$ [95\% CI 0.61-0.83] with $79 \%$ of calcium scores in concordant clinical categories, compared to standard CT-CAC. ${ }^{3}$ Future advancements in PET-CT scanner technology may allow calcium scoring to be performed using a helical image acquisition mode. This could potentially reduce the breath-hold artifacts, which are hypothesized to contribute to the small but statistically significant reductions in anterior wall perfusion. Iterative reconstruction techniques may further improve the quality of CTAC, especially in terms of noise and resolution characteristics. These reconstruction techniques are being introduced on current PET/CT systems and are becoming widely available for clinical use. Potentially these techniques could even further reduce the acquisition time, therefore limiting the risk for motion artifacts. Iterative $\mathrm{CT}$ reconstructions may also limit the effect of BMI on the CTAC image quality and therefore guarantee accurate $\mathrm{CAC}$ quantification, even for patients with higher BMI. On the other hand, one should also be aware of the possible impact of metal artifacts on CAC quantification, a pitfall that is also well known for standard CT-CAC quantification. However, new reconstruction techniques can also help avoiding this pitfall by reducing the metal artifacts using more accurate reconstruction models. ${ }^{4}$

The reduction of radiation dose that was achieved in this study is another important additional finding since the radiation burden to patients remains an issue of concern. In this context, a single MPI stress acquisition procedure especially in patients with low/intermediate risk on CAD will be another step forward, because the majority of the stress MPI findings will be normal. However, the value of a normal MPI needs to be projected against the complete assessment of all clinical variables obtained at the time of initial testing. ${ }^{5}$

Finally, accurate and reproducible CAC quantification can only be achieved by dedicated software, as also mentioned by the author, and by accurate calibration of the scoring methods. Therefore, improvements of the software in terms of visualization, automated detection, and user interaction will further enhance CAC quantification while accurate phantom calibrations can greatly improve plaque detection. ${ }^{6}$

\section{References}

1. Criqui MH, Denenberg JO, Ix JH, McClelland RL, Wassel CL, Rifkin DE, et al. Calcium density of coronary artery plaque and risk of incident cardiovascular events. JAMA. 2014;311:271-8.

2. Kaster T, Dwivedi G, Susser L, Renaud J, Beanlands RS, Chow BJ, et al. Single low-dose CT scan optimized for rest-stress PET attenuation correction and quantification of coronary artery calcium. J Nucl Cardiol. 2014. doi:10.1007/s12350-014-0026-y.

3. Mylonas I, Kazmi M, Fuller L, deKemp RA, Yam Y, Chen L, et al. Measuring coronary artery calcification using positron emission tomography-computed tomography attenuation correction images. Eur Heart J Cardiovasc Imaging. 2012;13:786-92.

4. Van SK, Nuyts J. Metal artifact reduction in computed tomography using local models in an image block-iterative scheme. Med Phys. 2012;39:7080-93.

5. Rozanski A, Gransar H, Min JK, Hayes SW, Friedman JD, Thomson LE, et al. Long-term mortality following normal exercise myocardial perfusion SPECT according to coronary disease risk factors. J Nucl Cardiol. 2014;21:341-50.

6. Arnold BA, Xiang P, Budoff MJ, Mao SS. Very small calcifications are detected and scored in the coronary arteries from small voxel MDCT images using a new automated/calibrated scoring method with statistical and patient specific plaque definitions. Int $\mathbf{J}$ Cardiovasc Imaging. 2012;28:1193-204. 